\title{
TRANSCUTANEOUS ELECTROSTIMULATION IN THE MANAGEMENT OF POSTOPERATIVE PAIN: INITIAL REPORT
}

\author{
S.B.C. BAKER, C.C. WONG, P.C. Wong and L.C. JeNkins
}

\begin{abstract}
A study was undertaken to investigate recent reports concerning the use of transcutaneous electrostimulation (TES) for relief of postoperative pain. Thirty patients undergoing elective herniorrhaphy were subjected to a standard perianaesthetic protocol. The patients were divided into three groups of ten, designated control, sham TES and TES. Postoperative analgesic requirements for each group were compared.

The number of intravenous doses of meperidine given to each group in the first three hours after operation was control group 46 doses; sham TES group 38 doses; TES group 10 doses $(p<0.0005)$. The number of intramuscular doses of meperidine in the 3-24-hour period was control group 21 doses; sham TES 22 doses; TES group 17 doses (no statistical difference between groups). A subjectively beneficial effect of TES was also established.

Despite a number of difficulties encountered during this study, primarily concerned with the subjective nature of pain and its assessment, a useful trend which warrants further investigation has been established in the use of transcutaneous electrostimulation (TES) for postoperative analgesia.
\end{abstract}

TransCUTANEOUS ElECTRICAL STIMULATION (TES) means passing an electric current through the skin surface through non-penetrating conducting electrodes. It is to be distinguished from percutaneous stimulation, which means current applied through electrodes such as needles, which penetrate the skin and from transcutaneous electrical nerve stimulation which implies a specific mechanism of action of the applied current through nerves.

TES for the management of chronic pain states was first reported by Shealy in 1972.' Since 1975, TES has been used in the Vancouver General Hospital Pain Clinic and has been found to be useful as a mode of therapy for the management of chronic pain. ${ }^{2}$ Conflicting reports have appeared in recent medical literature concerning the use of TES for relief of acute post-operative pain. ${ }^{3-5}$ This initial study was undertaken to evaluate the analgesic effects of TES in this type of pain in a clinical setting in the operation room and to determine whether it warranted a full scale trial on a larger number of postoperative patients.

TES has a number of advantages over traditional narcotic medications in the management of postoperative pain and, therefore, full evaluation of its potential is indicated. It does not depress

S.B.C. Baker, M.B., Ch.B.; C.C. Wong, M.B.B.S., M.R.C.P., F.R.C.P.(C); P.C. Wong, M.B., Ph.D.; L.C. Jenkins, M.D., C.M., F.R.C.P.(C). Department of Anaesthesia, University of British Columbia, Vancouver General Hospital, 855, 12th Avenue W., Vancouver, B.C., V5Z IM9.

Canad. Anaesth. Soc. J., vol. 27, no. 2, March 1980 the cardiovascular or respiratory systems and, apart from possible minor sedation, has no apparent effect on the sensorium; it is non-invasive, non-toxic, and relatively innocuous; it is simple in its application and can be used continuously or intermittently. Reduced incidence of atelectasis and ileus in the postoperative period have also been reported, but these remain unproved. ${ }^{6}$ Apart from contact dermatitis from the electrodes or conductive gel, complications and side effects have not been reported.

\section{METHODOLOGY}

\section{Patient selection}

Thirty patients of ASA status 1 or $2 *$ between 19 and 74 years of age who were undergoing elective herniorrhaphy were selected for this sludy. The patients were divided into three groups of ten, each group being comparable for age, sex, and duration of operation.

Group 1: A control group of ten patients was studied purely to establish baseline analgesic requirements for patients exposed to the perioperative protocol. They were not informed about the study, and they were not interviewed after the study.

Group 2: Sham TES. Group 3: TES. Patients in groups 2 and 3 were arbitrarily assigned to each group and differed only in the way TES was

*American Society of Anesthesiologists' assessment of physical status: ASA I = normal healthy patient. ASA $2=$ mild systemic disease. ${ }^{7}$ 


\section{TABLE I}

Evaluation of Post-Anaesthetic WAKEFULNESS

RECOVERY ROOM SCORE-MAXIMUM 6 (From STEWARD, D, J.)

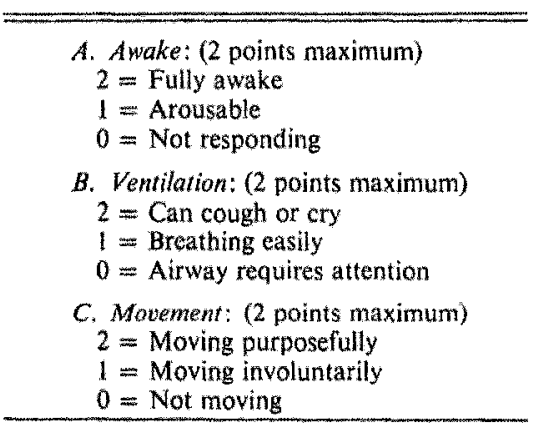

applied (see below). These patients were seen preoperatively by one of the investigators (SB). The study was fully explained to them, and a signed consent was obtained. Any questions concerning the study were answered, but they were not informed to which group they were being assigned.

In order to obtain ten patients in each group, 40 patients were studied. Six patients were excluded because they had a recovery room score of less than five (Table 1), three patients because bilateral procedures were carried out under the same anaesthetic and one patient because of severe unrelated postoperative back pain, resulting from previous traumatic lumbar spine compression fractures.

\section{Anaesthetic and postoperative protocol}

All patients received diazepam 5 to $10 \mathrm{mg}$ orally as premedication, followed by a general anaesthetic without the use of narcotic. Thiopentone was used for induction followed by the muscle relaxant of anaesthetist's choice and halothane, nitrous oxide and oxygen maintenance. After reversal of the muscle relaxant pa tients were transferred to the post-anaesthetic recovery area where they received oxygen until awake. Thirty minutes after operation each patient was assessed and assigned a recovery room score (Table I) by the recovery room nurses, to establish the degree of wakefulness, or recovery from the anaesthetic. Patients scoring less than five out of a possible six were not included in the final patient composition of the study.

Intravenous meperidine $5.0 \mathrm{mg}, 7.5 \mathrm{mg}$, or $10.0 \mathrm{mg}$, according to patient weight $(0.1 \pm$ $0.015 \mathrm{mg} \cdot \mathrm{kg}^{-1}$ ) was ordered for postoperative

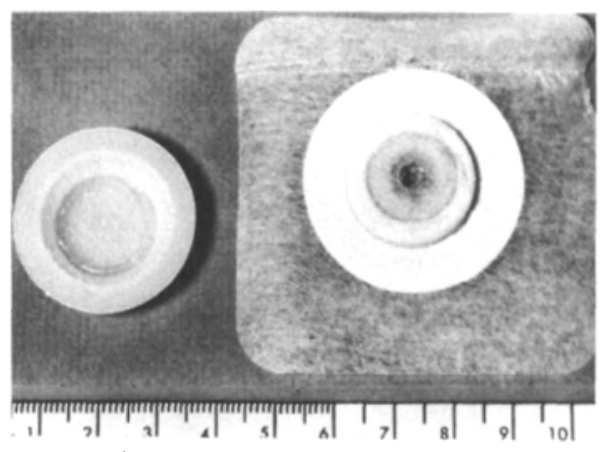

Ficure I Surface electrodes used for transcutane ous electrostimulation (patient contact area $1.76 \mathrm{~cm}^{2}$ ).

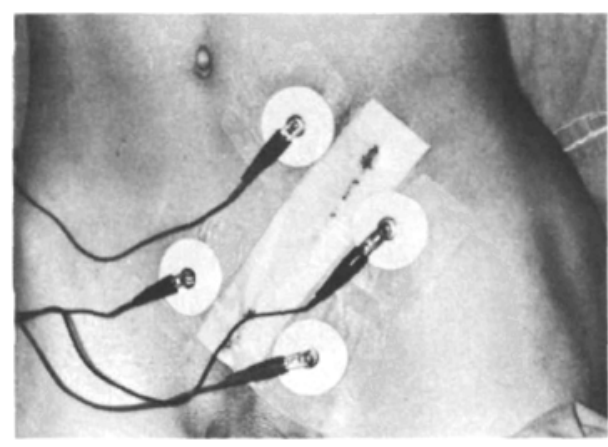

FIGURE 2 Positioning of stimulating electrodes on patient.

analgesia. Administration of the analgesic was left to the discretion of the nurses, at the patient's request. All patients received the usual monitoring and observations afforded to postoperative patients and remained in the recovery room for three hours, after which they were transferred to the ward. In the ward, intramuscular meperidine (approximately $1.0 \mathrm{mg} \cdot \mathrm{kg}^{-1}$ ) was given by the nurses for analgesia, at the patient's request.

\section{Method of stimulation}

Immediately after operation each patient in groups 2 and 3 had four standard adult electroencephalogram electrodes attached to the skin (Figure 1). Two were attached on either side of the herniorraphy incision approximately $3 \mathrm{~cm}$ from the incision and $5-8 \mathrm{~cm}$ apart (Figure 2), The electrodes were connected by insulated leads to an electrostimulator model $\mathrm{G} 6805$ made in Shanghai, China (Figure 3). The stimulator was modified to operate only on 4 " $D$ " type, 1.5 volt batteries. The characteristics of the stimulation were an asymmetric bipolar pulse of $1.0 \mathrm{mil}$ lisecond duration at a frequency of $50 \mathrm{Hertz}$ 


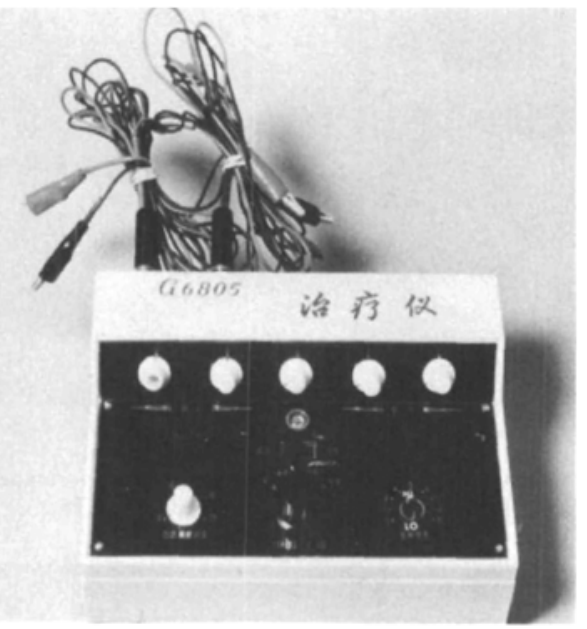

FIGURE 3 Electrostimulator-Model G6805.

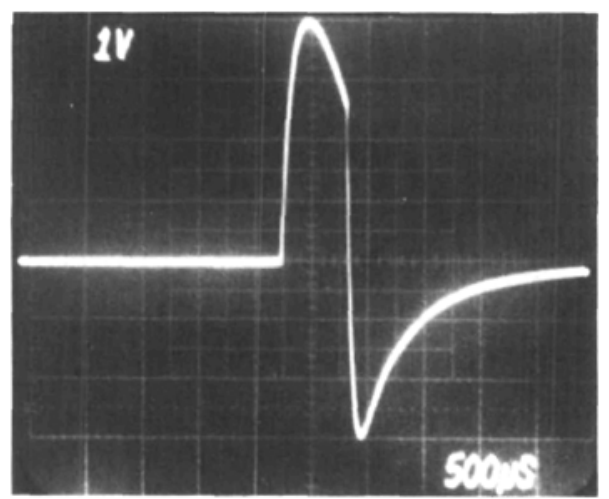

FIGURE 4 Wave form from Electrostimulator Model 66805 at average intensity of 4.0 volts (corresponds to amplitude dial setting of 2.0 ).

(Figure 4). The amplitude ranged from 3.0 to 6.0 volts peak to peak voltage. The current range was 3.0 to 6.0 milliamperes with an average skin resistence of $1000 \mathrm{ohms}$, which was well below the accepted fibrillation threshold of 75 milliamperes. ${ }^{\circ}$

In group 3, the TES group, the amplitude was preset at the minimum voltage until patients were awake, then it was increased until they were able to appreciate a comfortable tingling sensation.

In group 2, the sham TES group, altered leads were used so that the patient received no current despite the stimulating machine being switched on, and the amplitude dial was varied as in group 3 patients.

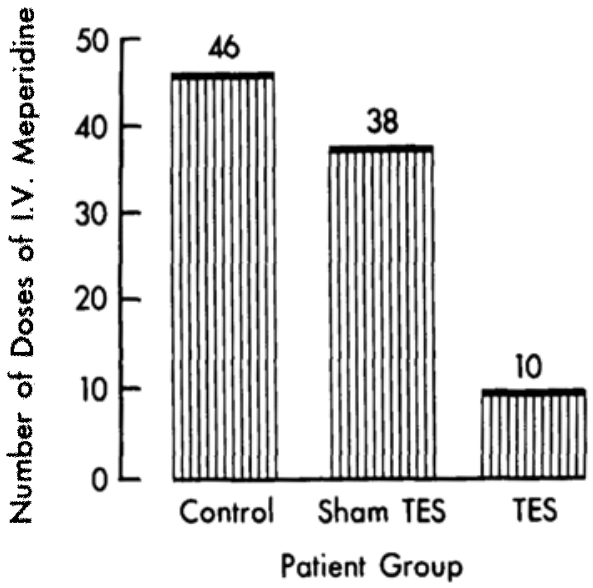

FIGURE 5 Analgesia administered in the recovery room. (First three hours postoperative.)

The TES and sham TES were discontinued after the three hours in the recovery room.

\section{ASSESSMENT}

Analgesic requirement for each patient for the first twenty-four postoperative hours was re. corded. The number of intravenous doses of meperidine given in the recovery room (up to three hours) and the number of intramuscular doses given in the ward (up to 24 hours) were totalled separately. A subjective assessment was requested from each patient and the nurses comments on any particularfeatures were noted.

\section{RESULTS}

\section{Analgesic requirements}

The number of intravenous doses of meperidine given to the patients in the three groups in the first three hours was: control group 46 doses, sham TES group 38 doses, TES group 10 doses (Figure 5). The TES group is significantly different from the control group ( $\mathrm{p}<$ $0.0005)$ and the sham TES group $(p<0.0025)$ when compared by the Student's t-test.

The number of intramuscular doses of meperidine given to the patients in the three- to twenty-four-hour period was: control group 21 doses, sham TES group 22 doses, TES group 17 doses (Figure 6). There is no statistical difference between these groups when compared by the Student's t-test.

The range of doses is shown by the individual 
TABLE II

Postoperative Analgesic ReQuirements

\begin{tabular}{|c|c|c|c|c|c|c|}
\hline \multicolumn{4}{|c|}{$\begin{array}{c}\text { Doses of I.V. meperidine } \\
0-3 \text { hours }\end{array}$} & \multicolumn{3}{|c|}{$\begin{array}{c}\text { Doses of I.M. meperidine } \\
3-24 \text { hours }\end{array}$} \\
\hline Patient & Control & Sham TES & TES & Control & Sham TES & TES \\
\hline 1 & 1 & 1 & 0 & 1 & 0 & 0 \\
\hline 2 & 1 & 1 & 0 & 1 & 4 & 0 \\
\hline 3 & 3 & 3 & 0 & 0 & 0 & 1 \\
\hline 4 & 3 & 3 & 0 & 4 & 1 & 3 \\
\hline 5 & 5 & 3 & 0 & 0 & 2 & 4 \\
\hline 6 & 5 & 3 & 1 & 3 & 3 & 1 \\
\hline 7 & 6 & 4 & 1 & 2 & 4 & 2 \\
\hline 8 & 6 & 5 & 2 & 2 & 3 & 3 \\
\hline 9 & 8 & 7 & 3 & 2 & 3 & 1 \\
\hline 10 & 8 & 8 & 3 & 6 & 2 & 2 \\
\hline TOTAL & 46 & 38 & 10 & 21 & 22 & 17 \\
\hline
\end{tabular}

Doses of meperidine administered to individual patients in the first twenty-four hours postoperatively. Patients tabulated for comparison according to increasing dosage requirement in the first three hours.

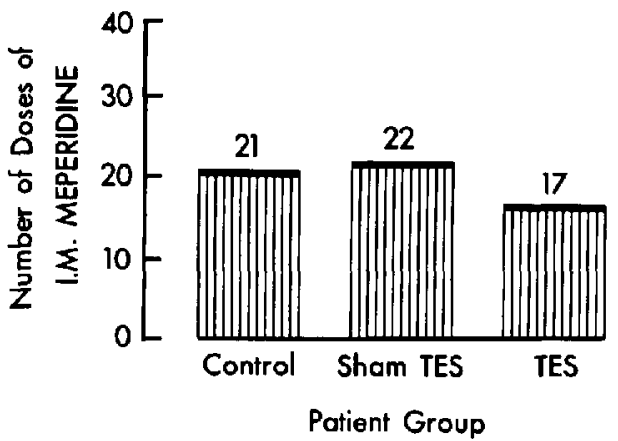

Figure 6 Analgesia administered in the ward (3-24 hours postoperative).

analgesic requirements of patients in the three groups in Table II.

\section{Subjective assessment}

In the TES group, eight patients felt that they had benefitted from TES and two could not decide because they lacked comparative incidents. Five of this group noted particularly that they had been given analgesic medication at night when they had no pain. Two patients recalled awakening from their anaesthetics with some pain but within 15 to 20 minutes of feeling TES they were completely comfortable

In the sham TES group, no patients felt that they received benefit. Eight patients stated that they did not receive benefit and two patients stated that (sham) TES had made their pain worse. The nurses noted that three of the patients were more restless than other patients from the study whom they had nursed. One patient who had had previous beneficial acupuncture experience felt disappointed that (sham) TES had not worked as he had anticipated. Another patient having sham TES recalled far more pain following this herniorrhaphy when compared with previous similar surgery.

\section{Discussion}

These results have shown that the analgesic requirement of patients in the TES group is considerably less than the control and sham TES groups in the first three hours after operation. The subjective assessment of the postoperative discomfort was also less in the TES group. The fact that five of the ten patients in the TES group required no analgesic medication in the three hour recovery room stay is an encouraging trend. If this could be duplicated in a group of respiratory or cardiovascular compromised patients, it would certainly be of benefit to them.

The analgesic effect of TES after the stimulation was discontinued in this study did not appear to be of sufficient duration to reduce the medication requirement significantly. It was the subjective impression of the interviewer (S.B.) that patients in the sham TES and TES groups had similar discomfort after the first one to two hours back in the ward. The value of continuing TES for longer than three hours to extend the analgesic effect needs to be studied further. 
Although the results appear significant in this small study, there were a number of factors which were not easily controllable. Pain is a subjective interpretation of nociceptive input, so that each patient will have a different appreciation of pain and pain threshold from equivalent surgical stimulus. Therefore individual analgesic requirements will vary considerably. The subjective interpretation of the patient's symptoms by the nurses administcring the analgesics create further variation. Until we can measure pain quantitatively this individual variation will remain a problem in any pain research.

Once the patients were fully awake the frequency of observations by the recovery room nurses tended to vary according to the nurses' workload and the amount of attention sought by the patients. The fact that the nurses and patients were participating in an experimental method which they were told might produce analgesia could have influenced the amount of analgesic drugs administered, particularly as they were all unaware that sham stimulation was being carried out. On the ward, again. frequency of patient contact by nurses and criteria for administering analgesics were extremely variable. On many occasions patients were given analgesics for sleep, or routinely following nocturnal recording of vital signs when, in fact, they had no pain. There is a need for more critical evaluation of the use of narcotic analgesics in postoperative pain management, particularly in the ward situation.

The mechanism of action of transcutaneous electrostimulation has not been completely clarified. Its action may be explained by modification of pain impulses ${ }^{10}$ at the spinal cord, midbrain or thalamic levels, or by release of endorphins which may initiate a central descending inhibitory mechanism. ${ }^{11,12}$

The exact role of placebo is difficult to assess and, although it may play a part, it is difficult to quantify.

\section{Conclusion}

Transcutaneous electrostimulation significantly reduced the immediate postoperative analgesic requirement in these patients undergoing herniorrhaphy. Further investigation of this mode of analgesic therapy is indicated to define more clearly its pattern of application and its role in the management of pain in the postoperative period.

A working study protocol has been established for use in future studies. The recovery room nurses have been familiarized with the equipment used and the surgeons, anaesthetists and nurses involved here accepted both the principle and the technique of transcutaneous electrostimulation.

\section{REFERENCES}

1. Shealy, C.N. Transcutaneous electroanalgesia. Surgical Forum 23: 419-21 (1972).

2. Wong. C.C., Wong, P.C. \& Jenkins. L.C. Report of Acupuncture Clinic for Pain, Vancouver General Hospital 1977; limited edition.

3. Hymes, A.C., RaAB, D.E. Yonehiro, E.G. NELSON, G.D. \& PRINTZ, A.L. Acute pain control by electrostimulation: a preliminary report. Advances in Neurology 4: 761-67 (1974).

4. Vanderark, G.D. \& McGrath, K.A. Transcutaneous electrical stimulation in treatment of post-operative pain. The Am. J. of Surg. Vol. 130, September 1975

5. PIKE, P.M.H. Transcutaneous electrical stimulation - its use in the management of postoperative pain. Anaesthesia 33: 165-171 (1978).

6. Hymes, A.C. Yonfhiro, E.G. RaAa, D.E. Nelson. G.D. \& Printz, A.L. Electrical surface stimulation for treatment and prevention of ileus and atelectasis. Surgical Forum 25: 222-224 (1974).

7. The American Society of Anesthesiologists House of Delegates' Document 1962.

8. STEWARD, D.J. A simplified scoring system for the post-operative recovery room. C.A.S.J. 22; 1 January (1975).

9. Wejbell, F.J. Electrical safety in the hospital. Ann. of Biomedical Engincering 2: 126-148 (1974).

10. Melzack, R. \& WALL, P.D. Pain mechanism: a new theory. Science 150: 971-979 (1965)

11. ZimMERMan, M. Neurophysiology of nociception (Abstract). Second World Congress on Pain 1: 173 (1978).

12. SJölund, B.H. \& ERIKsson, M.B.E. Endorphins and analgesia produced by peripheral conditioning stimulation (Abstract), Second World Congress on Pain 1: 15 (1978).

\section{RÉSUMÉ}

Cette étude porte sur l'évaluation du soulagement de la doulcur post-opératoire par l'électrostimulation transcutanée (EST). Trente malades opérés pour herniorraphie non urgente ont été soumis à un protocole anesthésique standard et divisés en trois groupes de dix désignés comme groupe contrôle, groupe EST fictif et EST. A la période post-opératoire, les besoins en analgésiques pour chaque groupe ont été comparés entre eux.

Le nombre d'injections de mépéridine administrées par la voie veineuse pour chacun des groupes pendant les trois premières heures qui suivaient l'opération a été de 46 pour le groupe 
contrôle, 38 pour le groupe EST fictif et de 10 pour le groupe EST $(p<0.0005)$. Le nombre de doses administrées par la voie intra-musculaire pendant la période s'étendant de 3 à 24 heures après l'opération a été de 21 doses pour le groupe-contrôle, 22 pour le groupe EST fictif et de 17 doses pour le groupe EST. Il n'y avait done pas pas de différence statistique entre chaque groupe. On a aussi établi que la stimulation électrique transcutanée avait un effet subjectif favorable.

Malgré les difficultés causées par la nature subjective de la douleur et son évaluation éprouvées durant cette étude, les auteurs croyent que la tendance favorable qu'ils ont démontrée justifie une évaluation plus poussée de la méthode. 